\title{
BLOOD PARASITES AFFECTS LOCAL RABBITS IN IRAQ,
} A REVIEW

\author{
Hind D. Hadi ${ }^{1}$, Zainab A. Makawi ${ }^{1}$, Farah A. Abedulmujeeb² \\ ${ }^{1}$ Iraq Natural History Research Center and Museum, University of Baghdad, Baghdad, Iraq \\ 2 Iraq National Cancer Research Center, University of Baghdad, Baghdad, Iraq \\ *Corresponding author: hinddhiaa86@gmail.com
}

\begin{abstract}
Rabbits are animals affected by many different species of parasites, infection Lead to lower body weight gain compared with non-infected rabbits, while sever infection Lead to death, although rabbits are less likely to develop epidemic diseases, but they are exposed to diseases of care and malnutrition, as well as parasitic diseases. Turning to previous studies that dealt with the spread of blood parasites in rabbits is a protozoan parasite such as (scab body, Babesia sp., Theileria sp., Anaplasma sp., Trypanosom sp. ,Plasmodium sp.). The current study aimed to defiend of blood parasite in rabbit in Iraq . Despite, the few of research on this subject for this study of blood parasites that Infection of rabbits and suggestion development of a database of studies of blood parasites affecting rabbits in Iraq.
\end{abstract}

Keywords: Rabbits, blood parasites, Babesia, Theileria, Anaplasma. 


\section{Introduction}

Rabbits are one of the most common laboratory animals, used around the world in Experiences to estimate various biological parameters, and this animal is either conventionally preserved in animal facilities for use in scientific research or is also obtainable in pet store $\mathrm{s}$ and in backyard settlements for commercial purposes (Pinto et al., 2004). Rabbits are susceptible to a number of parasites, among the parasitic diseases of rabbits, those which deserve special attention are coccidiosis, skin mange, ear mange or ear cancer and stomach-worm diseases. The common results are emaciation, weakness, wasting and death ,other parasitic affections, Such as irritation caused by fleas, lice and intestinal worms of different types, although no noticeable symptoms are common in infected animals, they may gradually make them weak and unthrifty, making them more susceptible to other diseases due to their low vitality (Schwartz and Shook, 1931). The

\section{Babesia species:}

Babesia is a genus of parasites that belongs to the phylum Apicomplexa, Babesiosis is transmitted through the bite of infected Ixodes ticks, by blood transfusion or congenitally. In spite of fever is a hallmark of babesiosis, there are some no specified symptoms (Vannier et al. 2015). The disease mostly occurs in the USA, but cases have also been reported in several European countries, in India, Egypt, Korea, Taiwan, and South Africa (Nassar and Richter, 2017). The main pathological event is lyses of Red blood cells lead to hemolytic anaemia which in heavy cases may lead to organ failure and death, (Gray et al., 2010). internal parasites of rabbits include very fine organisms known as protozoa and such tapeworms and round worms. They can cause loss of condition and ill health in rabbits as well as posing a risk to people. Most pets have these, although it's not always easy to tell if your rabbit is infested as often there aren't any outward signs of ill health. Symptoms are difficult to spot, but can include loss of condition, vomiting and diarrhea. By the time you notice these symptoms, these parasites can be damaging your rabbit's health. (Patton et al., 2008). As for the blood parasites of rabbits primary organisms that live and feed on animal blood where they are found within RBCs and WBCs or in plasma between blood cells and pose a significant risk to their life If the correct diagnosis and treatment are not made (Ambrosio and Dewaa, 1990). The most important of which are Babesia Theileria , Plasmodium , Anaplasma and Trypanosome.

\section{Theileria species :}

Theileria is a genus of parasites belonging to the group Apicomplexa, and is closely related to Plasmodium. Theileria affects a variety of domestic and wild animals and is transmitted by tick ixodid from the genera Amblyomma Haemaphysalis, Hyalomma and Rhipicephalus. Most of these ticks are famous for its large economic losses caused by the agricultural industry because of the spread of diseases and deaths and leathers damage and poor pet production. (Bishop et al., 2004). The amplification and conservation of wildlife has make Theileria of wildlife important theme for study. Theileria can be categorized into "conversion" and "non-conversion" factions. Parasite conversion of all groups in T. taurotragi clade (Sivakumar et al., 2014), uncontrolled 
spread of schizonts results in diseases related with Corridor disease (Theileria parva), East Coast fever (T. parva), malignant theileriosis (T. lestoquardi) in goats and sheep (Bishop et al., 2004; McKeever, 2009). While, T. sp. (bougasvlei), T. taurotragi, and T. sp. (buffalo) does not lead to diseases associated with schizot (Bishop et al., 2004). Theileria sp. that group within the antelope Theileria causes lymphatic hypertrophy commonly related with the conversion of Theileria (Nijhof et al., 2005). This and the ability to culture schizonts indicate that not all the parasites converted share a monophyletic origin, which propose that schizonts transformation may happen on a larger scale than expected. The non- conversion Theileria are benign but it can still cause infection due to plasma anemia induced by the piroplasm stage (Sivakumar et al.,2014).

\section{Plasmodium species:}

Plasmodium falciparum (Welch, 1897) is more famous in the tropics and subtropics area, stages of $P$. falciparum in the human host are (1) entering sporozoite (2) pre-erythrocytic schizont (3) asexual forms in the red blood cells and (4) gametocytes developing in the red blood cells . (P. ovale, P.vivax, , P. falciparum , P.malariae) reproduce asexually in man and sexually in Anopheles mosquitoes .The infected mosquito injects sporozoites that enter liver parenchymal cell and undergo pre-erythrocytic schizogony to produce numerous merozoites. Merozoites released into the blood stream enter erythrocytes and undergo schizogony to produce more merozoite (Obado et al., 2016). Asexual life cycle in red blood cells generally needs one to two days in P.falciparium ,two days in P.vivax and P. ovale. and three days in P. malariae. Some merozoites that enter erythrocytes develop into macro and micro gametocytes that will initiate the sexual cycle if ingested by susceptible mosquito. Malarial infection can be acquired by blood transfusion ,congenitally by organ transplant , and by the shared use of contaminated needles by intravenous drug users (Coatney et al., 1971). Pathology is primarily due to the erythrocytic stages and involves not only the red blood cells but also the spleen, liver, and other visceral organs in P.falciparum (Frederick ,2002).Malaria changes in the surface of the infected erythrocyte lead to hemostasis in the blood sinuses capillaries particularly in the brain, lung , coronary vessels and kidney (Bartoloni and Zammarchi, 2012).

\section{Anaplasma species:}

Anaplasma is one of the diseases of infectious blood parasites and is spread primarily by ticks and is not transmitted in direct contiguity. Hot and underground areas. Anaplasmosis is also transmitted by the Ixodes tick which typically transmits Lyme disease (Yabsley et al., 2006). Anaplasmosis is known as a disease spread by ticks. This can be contracted from tick bites that contain anaplasma phagocytosis. The most common bacterial tick that has bacteria is known as black-legged tick. This can be done by common deer tick. Anaplasmosis can also be transmitted by blood transfusion, if the patient has contracted the disease unknowingly, then his blood is released. The infection can also be contracted by the recipient. It may also be spread through the use of unmodified surgical instruments, dehydration, castration, tattoo and needles under the skin. (Capucille, 2011). 


\section{Trypanosomes species:}

Wide range of blood parasites, which cause trypanosomiasis in both animals and humans (Merino Espinosa et al., 2016) like T.vivaxs, T.bruce ,T.rhodesiense, T. gambiense, T.congolense and T. evansi. Trypanozoon evansi was first diagnosed and named by 'Griffith Evans' in 1880 in Punjab / India by infected horses and camels, causing illness known as (surra). In Iraq T.evansi first diagnosed by pioneer Chadwick (1938) in the dogs (Hoare., 1956). and in camels as an enzootic disease. Trypanosome evansi also affected buffalo and cattle (Leiper, 1956). In humans, trypanosomiasis is known as African sleeping sickness. The main way of spreading these parasites is by a mechanical transmission. In another meaning, a kind of haematophagous insects, such as, Tobanid flies can transfer infected blood to other healthy organisms. Trypanosome species carried by Tobanid flies, T.evansi remains monomorphic throughout its life cycle, while $\mathrm{T}$. brucei subspecies presents different set of shapes during different points of her life cycle (Hoare., 1972).

\section{Encephalitozoon cuniculi :}

Encephalitozoon cuniculi One of the microscopic parasites infecting the renal and central nervous system and causing infection to animals such as rabbits (Oryctolagus cuniculus). (Deplazes et al., 1996). It is a major disease of captive rabbits and has international recognition of its seroprevalence. Most physicians mention vestibular disorder as one of the most frequently reported clinical signs. releated with chronic E. cuniculi infection. One of the most common species of the Encephalitozoon genus and the phylum microsporidia genus is cuniculi (Keeblem and Shaw 2006). Although a comprehensive review of the literature detects E-caused lesions. Cuniculi are usually found in the central nervous system (CNS) and these lesions are not consistent with clinical signs reported. The encephalitozoonosis pathogenesis and life cycle makes E. Cuniculi escaping the serological titer association with the extent of the clinical disease, lacking humoral-mediated immunity in their rabbit host, remain unaffected by therapeutic attempts to reduce sporontaneous inflammation and Escape therapeutic attempts in patients with chronic infection to eradicate germination, As E. Cuniculi has been involved in immunocompromised humans as an opportunistic zoonotic agent, it becomes necessary to realize its true prevalence in the pet trade Disease control and reduction of transmission are critical for veterinary and public health (Mathis et al.,2005).

\section{Review in the previous studies Distribution of blood parasites from Rabbits}

In Iraq there are very few studies on this subject there are only two studies:First study Abdulmajeed et al., (2007) in their study for some hematological and pathological changes in blood and liver of adult male rabbits experimentally infected with Iraqi strain of Trypanosome evansi they found the infection induced clinical symptoms of disease presented as acute and chronic phases depending on the duration of infection, Leucocytosis due to marked lymphocytosis identified in the acute phase come after leucopenia through chronic phase. The major differences in the RBCs were the presence of 
large-size cells, howell-Jolly bodies, sperm cells, target cells ,Burr cells, and a significant platelets deficiency (Thrombocytopenia) were monitored. The second study Athraa, (2014) who study the prevalence of gastrointestinal and blood parasites with some histopathological changes of local rabbits in Baghdad province, who found some species of blood protozoa that infect local rabbits, that the total infection rate of blood protozoa (38\%) included (Thieleria, Babesia, Anaplasma and Plasmodium) in the percentage $(23.75,5,6.25$ and 25) respectively. Dipeolu and Ogunji (1977). In their study of the transfer of Theileria annulata to a rabbit by the larvae of the tick Hyalomma rufipes they found a white rabbit from New Zealand on which larvae of Hyalomma rufipes were fed died of theileriosis because of Theileria annulata 22 days after the larvae had fed rufipes since its blood was free of Theileria parasites before the larvae were fed ; .Moreover, The blood of the rest of the rabbits rabbits, on which the nymphs and adults were feeding, was free of the Theileria parasite .

Goethert and Telford (2003) They recorded the rate of rabbits infected with Babesia ranged from $11 \%$ to $29 \%$, were examined over five transmission seasons. Heidi and Sam (2003).While studying Enzootic Transmission of Anaplasma bovis in Nantucket Cotton tail Rabbits They found that the prevalence of $A$. bovis in cotton tail rabbits ranged from 4 to $32 \%$ with a total average of $18 \%$ and They accounted for differences in prevalence that were not significant when evaluated on an annual basis but were significant when evaluated on a monthly basis.Holman et al., (2005) The researchers reached through their study of isolating the Babesia sp. from Eastern Cottontail Rabbits that the nine samples were positive for babesias by PCR (88 total, 10.2\% prevalence) during 2002 and three samples were positive (26 total, $11.5 \%$ prevalence) in 2003. Yabsley et al., (2006). The researchers carried out a molecular survey of several tickborne pathogens in three types of rabbits To reveal Anaplasma and Babesia parasites in rabbits from Georgia, USA and Texas these results indicate the rate of rabbits infected with Babesia (28\%) and A. bovis (61\%). Qing et al., (2015) Researchers, through their study of serological spread of Encephalitozoon cuniculi and Toxoplasma gondii in local rabbits (Oryctolagus cuniculus), found that E. cuniculi is found in rabbits on a large scale. The Information about E. cuniculi serological spread is important not only because of on the animals potential from this parasite but also because the parasites can cause significant economic losses due to animal deaths of the hosts.

\section{Conclusion}

Rabbits have many parasites, including the hematomas, which lead to transitional diseases that affect humans and animals.The danger lies in its presence within RBCs and WBCs or in plasma between blood cells. Therefore, the study focused on investigating the parasites that infect rabbits, including blood.

\section{Conflict of interest}

The authors declare no conflicts of interest. 


\section{References:}

- Abdul-majeed, H. A. Abbas, F. H. and Abbood, H. F. (2007). Blood picture and hepatic changes in rabbits experimentally infected with Trypanosoma evansi. Iraqi strain. Medical Journal of Babylon ; 4 (3):358-364.

- Ambrosio, R. E. and Dewaal , D .T . (1990). Diagnosis of parasitic disease Rev. sci. tech. Off. int. Epiz ; 9 (3): 759-778.

- Athraa, T. W. (2014). Study of Prevalence of gastrointestinal and blood parasites with some histopathological changes of local rabbits in Baghdad province. M.Sc. Thesis, College of Veterinary Medicine, University of Baghdad.

- Bartoloni, A. and Zammarchi, L. (2012). Clinical aspects of uncomplicated and severe malaria. Mediterranean Journal of Hematology and Infectious Diseases; 4 (1): e2012026

- Bishop, R. Musoke, A. Morzaria, S. Gardner, M. and Nene, V. (2004). Theileria: intracellular protozoan parasites of wild and domestic ruminants transmitted by ixodid ticks. Parasitology; 129, 271-283

- Capucille, D. J. (2011). "Anaplasmosis". In Haskell, SRR (ed.). Blackwell's Five-Minute Veterinary Consult Ruminant. Hoboken: John Wiley \& Sons. pp. 50-51.

- Coatney, G.R. Collins, W.E. Warren, M. and Contacos, P.G. (1971) .The Primate Malarias. U.S. Department of Health, Education and Welfare, Bethesda ;64-76.

- Deplazes, P. Mathis, A. Baumgartner, R. Tanner, I. and Weber, R. (1996. Immunologic and molecular characteristics of encephalitozoon-like microsporidia isolated from humans and rabbits indicate that Encephalitozoon cuniculi is a zoonotic parasite. Clin Infect Dis; 22(3):557-559.

- Dipeolu, O. O. and Ogunji, F. O. (1977). The transmission of Theileria annulata to a rabbit by the larvae of the tick Hyalomma rufipes. Laboratory Animals; 11, 39.40.

- Frederick, L. S.(2002). Cultivation of Plasmodium spp. Clinical Microbiology Reviews, 15(3). 355-364.

- Heldi, K. G. and Sam, R. T. (2003). Enzootic transmission of Babesia divergens among cottontail rabbits on Nantucket Island ,Massachusetts. Am. J. Trop. Med. Hyg; 69(5): 455-460.

- Goethert, H. K. Telford, S.R. (2003). Enzootic Transmission Of Babesia Divergens Among Cottontail Rabbits On Nantucket Island, Massachusetts . Am. J. Trop. Med. Hyg .69(5). pp. 455-460.
- Gray, J. Zintl, A. Hildebrandt, A. Hunfeld, K. and Weiss, L. (2010). Mini Review Zoonotic babesiosis: Overview of the disease and novelaspects of pathogenidentity. Ticks and Tick-borne Diseases; 1, 3-10.

- Hoare, C. A. (1956). Morphology and Taxonomic Studies on Mammalian Trypanosomes. VIII. Revision of Trypanosoma evansi. Parasitology; 46, 130-172.

- Hoare, C. A. (1972).The Trypanosomes of Mammals, Blackwell Scientific Publications. Oxford. pp.447.

- Holman, A. M. Spencer, R. E. Heidi, K. G. and Samuel, R.T. (2005). In Vitro Cultivation of a Zoonotic Babesia sp. Isolated from Eastern Cottontail Rabbits (Sylvilagus floridanus) on Nantucket Island, Massachusetts . Journal of Clinical Microbiology ; 43 (8):3995-4001.

- Keeblem, E. and Shaw, J. (2006). Seroprevalence of antibodies to Encephalitozoon cuniculi in domestic rabbits in the United Kingdom. The veterinary record 158, 539-544.

- Leiper, J. W. G. (1956) . Report to the Government of Iraq on animal parasites and their control, F.A.O., No; 610.

- Mathis, A. Weber, R. and Deplazes, P. (2005). Zoonotic potential of the microsporidia. Clin Microbiol Rev 18(3), 423445.

- McKeever, D. J. (2009). Bovine immunity - a driver for diversity in parasites? Trends Parasitol. 25,269-276.

- Merino Espinosa, G. Corpas López, V.Morillas -Márquez, F. and .Díaz-Sáez,V.(2016). Genetic variability and infective ability of the rabbit trypanosome, Trypanosomanabiasi Railliet 1895, in southern Spain.Infection, Genetics and Evolution 45, 98-104.

- Nassar, Y. and Richter, S. (2017). Babesiosis Presenting as Acute Liver Failure. Case Rep Gastroenterol; 11(3): 769-773.

- Nijhof, A. M. Pillay, V. Steyl, J. Prozesky, L. Stoltsz, W. H.and Lawrence, J. A. (2005). Molecular characterization of Theileria species associated with mortality in four species of African antelopes. J. Clin. Microbiol; 43,59075911.

- Obado, S. O. Glover, L . and Deitsch, K.W. (2016). The nuclear envelope and gene organization in parasitic protozoa: Specializations associated with disease . Molecular and Biochemical Parasitology; 209 (1-2): 104-113. 
- Patton, N. M. Hagen, K. W. Gorham, J. R. and Flatt, R. E. (2008). Domestic Rabbits Diseases and Parasites: APacific Northwest Extension Publication. P 19-24.

- Pinto, R.M .Gomes, D.C. Menezes, R.C. Gomes, C. T. and Noronha, D. (2004). Helminths of rabbits (Lagomorpha: Leporidae) deposited in the Helminthological collection of the Oswaldo Cruz institute .Revista Brasileira de Zoologia ;21(3): 599-604.

- Qing, F. M. Wei, L. W. Xiao, T.N. Hai, B. L. Gui, Z. Y. Xiao, L. S. Wei, L. W. and Wei, C.(2015).Seroprevalence of Encephalitozoon cuniculi and Toxoplasma gondii in domestic rabbits (Oryctolagus cuniculus) in China. Korean J Parasitol; 53(6): 759-763.
- Schwartz, B. and Shook, W.B.(1931). Rabbits Parasites and Diseases. U.S. Department of agriculture, Farmers' Bulletin NO. 1568, Issued, 1928. Slightly revised; $\mathrm{p}: 1-24$.

- Sivakumar, T. Hayashida, K. Sugimoto, C. and Yokoyama, N. (2014). Evolution and genetic diversity of Theileria. Infect. Genet. Evol 27,250-263.

- Vannier, E.G . Diuk-Wasser, M .A. Ben, M.C. and Krause, P.J. (2015). Babesiosis. Infect Dis Clin North Am 29,357-370.

- Yabsley, M.J. Romines, J. and Nettles, V.F. (2006). Detection of Babesia and Anaplasma species in rabbits from Texas and Georgia, USA. Vector Borne Zoonotic Dis ; 6(1):7-13. 